Absorption spectra are produced using a linear ramp generator to create a frequency variation. Because the positions of the iodine absorption lines are well known [6], a scaling of the frequency axis is possible. Variations in the line shapes due to temperature fluctuations $(\Delta T \leq 0.1 \mathrm{~K})$ are not significant [7] for the conditions presented here. Using the absorption spectrum the laser light tuning coefficient for our laser was estimated to be $1.510 \mathrm{GHz} / \mathrm{V}$

As can be seen from Fig. 2, near the low frequency turning point of absorption line 1104 , a light intensity variation of $1 \%$ indicates a laser frequency variation of $6.95 \mathrm{MHz}$. By observing the light intensity passing the iodine cell, frequency drift measurements can be made. For example, about $30 \mathrm{~min}$ after switching on the free running laser, the frequency shows a drift rate of $450 \mathrm{kHz} / \mathrm{min}$.

Fig. $3 a$ illustrates the operational mode of the automatic frequency control (AFC) after switching on the laser. The frequency deviation which is shown as a function of time is
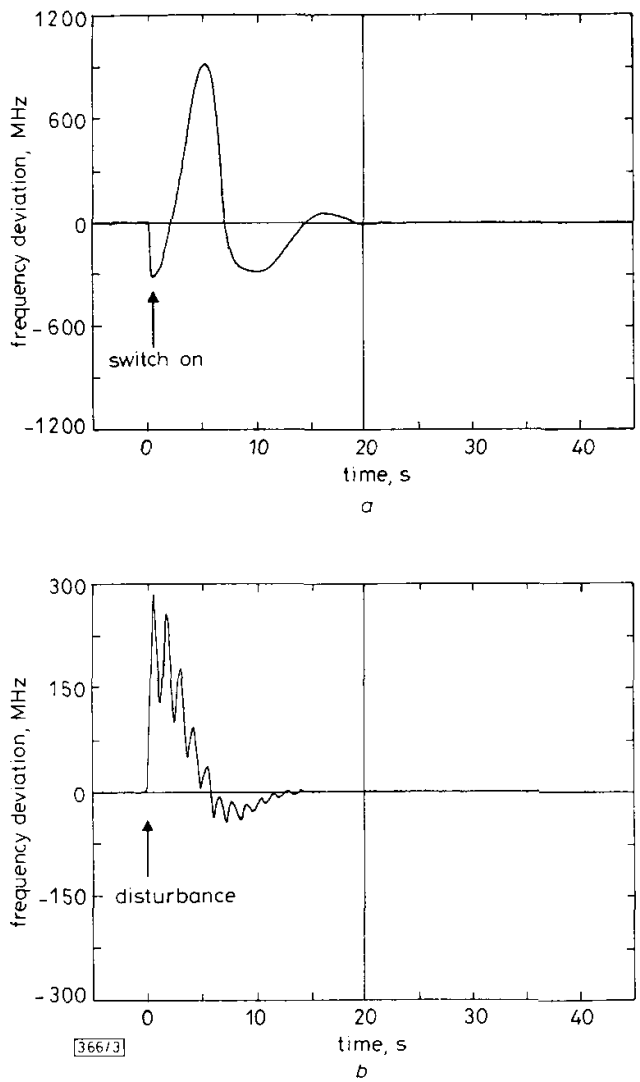

Fig. 3 Laser frequency deviation with respect to time (frequency search process)

a After laser is switched on

$b$ After an externally induced frequency disturbance

proportional to the voltage measured at the temperature control input, at low response times $(\sim 1 s)$. The initial frequency of the laser was set about $1 \mathrm{GHz}$ below where the frequency of the laser should be locked. About $20 \mathrm{~s}$ after switch on, the frequency is then locked. The remaining frequency jitter was measured to be less than $17 \mathrm{MHz}$, i.e. relative frequency stability $\Delta f / f \geq 6 \times 10^{-8}$ has been achieved.

Fig. $3 b$ shows the frequency response of a locked laser after a disturbance which was induced externally by a voltage pulse directed into the temperature control input. A frequency deviation of $\sim 300 \mathrm{MHz}$ is compensated for within $15 \mathrm{~s}$. The superposed oscillation, having a frequency of $0.8 \mathrm{~Hz}$, arises from the internal temperature control of the laser.
It was found that the locking frequency should be fixed, or be close to the turning point of an absorption line, because there, the slope of the flank in addition to the capture range of the AFC is as large as possible. Considering calibration problems $[6,7]$, inaccuracy of the measuring instruments, external electrical disturbances etc., the laser frequency can be determined and reproduced with an absolute accuracy of $\sim 30 \mathrm{MHz}$, translating to $\Delta f / f \simeq 10^{-7}$. To accurately measure the absolute frequency stability, two completely independent locking systems need to be built, because their heterodyne beat signal observed with an RF spectrum analyser directly shows their frequency behaviour.

Conclusion: In summary, a simple method of frequency locking of a diode pumped $\mathrm{Nd}$ : YAG laser to an iodine absorption line has been presented. Because the concept can be realised in a relatively robust, compact, light and inexpensive arrangement, application to coherent free-space communication or lidar systems should be possible. To test the method presented here, in conjunction with other principles of frequency changing (piezoelectric transducer or light induced thermal frequency variation [8]), further investigations are in progress.

Acknowledgments: The authors wish to thank Mr. Becker (University of Mainz, Germany) for providing the iodine cell.

C IEE 1993

17th March 1993

R. Heilmann and J. Kuschel (German Aerospace Research Establishment (DLR), Institute of Optoelectronics, D-8031, Oberpfaffenhofen Germany)

References

1 Wallmeroth, K., Wandernoth, B., franz, J., Meier, H., and SCHORP, B.: 'Towards a coherent optical free-space communication system', Electron. Lett., 1990, 26, pp. 572-573

2 KANE, T. K. NILSSON, A. C., and BYER, R. L.: 'Frequency stability and offset locking of a laser-diode-pumped Nd : YAG monolithic nonplanar ring oscillator', Opt. Lett., 1987, 12, pp. 175-177

3 planar ring 0scillator', Opt. Lett., 1987, 12, pp. 175-177 for lasers at $\lambda=1.06 \mu \mathrm{m}$ ', Opt. Lett., 1990, 15, pp. 812-813

4 ARIE, A., SCHILLER, S., GUSTAFSON, E. K., and BYER, R. L.: 'Absolute frequency stabilisation of diode-laser-pumped Nd: YAG lasers to hyperfine transitions in moleculare iodine', Opt. Lett., 1992, 17, pp. 1204-1206

5 PRIBIL, $K$, JOHANN, $\mathrm{U}$, and SONTAG, $\mathrm{H}$ : 'SOLACOS: a diodepumped Nd: YAG laser breadboard for coherent space communication system verification'. Proc. SPIE, 1991, 1522, pp. 36-41

6 GERSTENKORN, S., and LUC, P.: 'Atlas du spectre d'absorption de la molecule d'iode' (Laboratoire Aimé Cotton, Orsay, France, 1980)

7 SHimoda, K. (Ed.): 'High-resolution laser spectroscopy' (SpringerVerlag, Berlin, Heidelberg, New York, 1976)

8 HEILMANN, R., and WANDERNOTH, B.: 'Active light induced therma frequency stabilisation of monolithic integrated twisted-modecavity Nd : YAG laser', Electron. Lett., 1992, 28, pp. 1367-1368

\section{TESTING OF MIXED-SIGNAL SYSTEMS USING DYNAMIC STIMULI}

D. Taylor, P. S. A. Evans and T. I. Pritchard

Indexing terms: Integrated circuits, Testing

The impulse response of a linear circuit element contain enough information to functionally characterise that element. A technique for comparison of observed and expected Areference) transient responses, which results in an absolute measure of device functionality, is presented. Comparisons or the results from existing test programs are also presented.

Introduction: Mixed-signal testing is a complex subject since it encompasses all of the problems associated with digital testing, all of the problems associated with analogue testing 
and some additional problems which are specific to mixedsignal testing and involve the internal connections between the analogue and digital domains. The inclusion of an interface scan (IS) path [1] partitions the analogue and digital test problems and provides controllability and observability of the internal analogue/digital interface. Assuming that the digital sections of a circuit can then be tested using existing test techniques the mixed-signal test problem reduces to an analogue test problem.

Transient response testing (TRT) [2-4] has been identified as a powerful technique for testing linear circuit elements. This technique is particularly useful in mixed-signal testing because the simple logic amplitude stimuli employed can be propagated through digital circuitry or injected via the interface scan path.

This Letter describes a technique for the automatic comparison of observed and reference transient responses and the generation of an absolute measurement of similarity, termed the index of functionality $I_{F}$

Comparison of observed and reference transient responses: The analysis of transient responses employs a correlation technique which means that sample rates and intervals can be inferior to those dictated by the Nyquist criteria.

If $X_{n}$ is an array of $n$ evenly spaced data samples from an observed transient response at time intervals $\tau$, and $Y_{n}$ is a se of similar data samples taken from a reference (expected) transient response (either simulated or from a 'golden' device) the crosscorrelation function $R_{x y}$ is formed by

$$
R_{x y}=\frac{1}{n} \sum_{K=1}^{n} x(K \Delta t-\tau) y(K \Delta t)
$$

and the autocorrelation function $R_{y y}$ is formed by

$$
R_{y y}=\frac{1}{n} \sum_{K=1}^{n} y(K \Delta t-\tau) y(K \Delta t)
$$

The index of functionality is then given by comparing $R_{x y}$ with $R_{y y}$ :

$$
I_{F}=\left(1-\frac{1}{n \zeta} \sum_{K=1}^{n}\left|R_{x y}-R_{y y}\right|\right) \times 100 \%
$$

where $\zeta$ is a figure computed to normalise the index of functionality.

The constant $\zeta$ is derived from a comparison of the reference response and the 'most faulty' response which might reasonably be observed from a device under test. Under normal circumstances this will be a response where the output is permanently stuck-at one of the supply rails. A device which exhibits a perfect (reference-like) transient response will therefore generate an index of functionality of $100 \%$ and a device with a catastrophic fault will generate an index of functionality approaching $0 \%$.

Having arrived at this absolute measurement of functionality, the problem is then in defining the limit, $I_{F L}$, for passing and failing devices, where $100 \%>0 \%$. By simulating functional faults which take the response of a device outside the limits defined by its specification the effect of such faults can be mapped into the index of functionality. Simulations have shown that this limit will usually be in the range $90-99 \%$ Therefore if the response from a device under test produces an index of functionality $I_{F} \geq I_{F L}$ that device is said to be functional and if a device response produces an index of functionality $I_{F}<I_{F L}$ that device is defined as faulty

Comparison of transient response tests with conventional tests: As part of a DTI/SERC LINK project involving GEC Plessey Semiconductors, Wolfson Microelectronics, the University of Huddersfield and UMIST, a mixed-signal test vehicle (MSTV) has been developed and fabricated. In addition to digital and analogue functions this device features an interface scan for control of the digital/analogue interface. The results presented here concentrate on an 8 bit $D / A$ convertor which was one subsystem of the MSTV, the output of which can be treated as linear with the test sequence employed.
A production run of 200 devices was used as a reasonable statistical sample. Over and above simple pass/fail measurements the results for the $\mathrm{D} / \mathrm{A}$ convertors were datalogged by GEC Plessey and the performance of each device assessed in relation to the rest of the sample. These results included measurements of linearity, gain and DC supply parameters.

The D/A convertors were tested using a transient response test, and the results compared to those obtained using a conventional test program. The transient response test involved accessing the input of the D/A convertor via the interface scan path, clearing it then propagating a stream of logic ' 1 's through the input lines. This test sequence was designed to test the linearity and gain of the device, and for the presence of stuck-at or bridging faults on the digital input lines.

Shown in Fig. 1 are the ordered index of functionality results from the transient response tests of $200 \mathrm{D} / \mathrm{A}$ convertors. The devices towards the origin of the $x$-axis with an

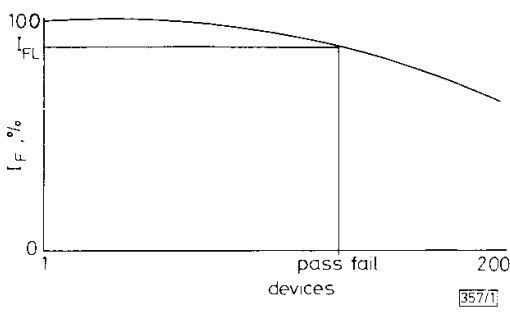

Fig. $1200 \mathrm{D} / \mathrm{A}$ convertors ordered in decreasing index of functionality

index of functionality approaching $100 \%$ are good devices, and the devices towards the right of the graph are faulty devices with reduced index of functionality. However, the most faulty device in the sample of 200 generates an index of functionality of $70 \%$. This is because the normalised index of functionality is heavily dependent on the DC level of the reference response. For this particular test, a device where the output node is stuck at the positive supply rail would generate an index of functionality of $0 \%$

In this case the limit $I_{F L}$ is set at $92 \%$ and the pass/fail decisions are shown in Fig. 2. The devices highlighted on the

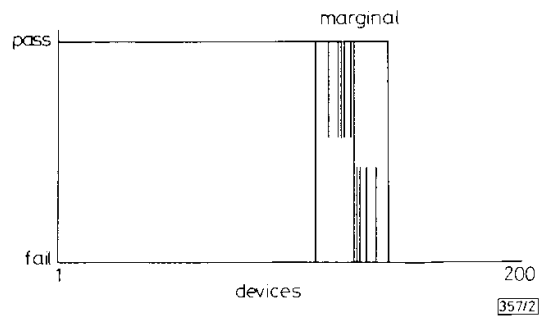

Fig. 2 Pass/fail decisions and discrepancies with conventional test program

graph are devices where the results of the transient response test disagree with the results of the conventional test program. In total there are nine such devices all clustered around the limit $I_{F L}$

Conclusions: This work demonstrates that the results of the transient response tests are in close agreement with the results of the conventional test program. The discrepancies around the limit $I_{F L}$ are tolerable and can be attributed to devices with marginal functionality, and therefore marginal responses. The results from the 40 devices within $+10 \%$ of the limit $I_{F L}$ are in fact so similar as to make ordering meaningless.

However, the most significant aspect of these results is that the conventional test program would run on a $£ 1 \mathrm{M}$ LTX tester whereas the transient response tests were performed using a $£ 15 \mathrm{~K}$ digitising oscilloscope and a $486 \mathrm{PC}$. Transient response testing, as it stands, can be used as a quick and inexpensive wafer probe test prior to packaging, however the

ELECTRONICS LETTERS 29th April 1993 Vol.29 No. 9 
next phase of this work is to further develop and characterise transient analysis into an accurate production test technique.

(C) IEE 1993

18th March 1993

D. Taylor, P. S. A. Evans and T. I. Pritchard (Division of Electronics \& Communications, University of Huddersfield, Queensgate, Huddersfield HDI 3DH, United Kingdom

\section{References}

1 PRITCHARD, T. I., EVANS, P. S. A., and TAYLOR, D.: 'The development of generic testing strategies for mixed-signal ICs', IEE Proc. $G$ April 1992, 139, (2)

2 EVANS, P. S. A., AL-QUTAYRI, M. A., and SHEPHERD, P. R.: 'A novel technique for testing mixed-signal IC's'. Proc. ETC, April 1991 Munich, Germany

3 TAYLOR, D., EVANS, P. S. A., and PRITChaRd, T. I.: "Transient response testing of mixed-signal ASICs'. Proc. IEEE ASIC Conf. September 1992, Rochester, New York

4 TAYLOR, D. EVANS, P. S. A, and PRITCHARD, T. L. 'A comparison of DC and transient responses tests for analogue circuits'. Proc ESSCIRC, September 1992, Copenhagen, Denmark

\section{REALISTIC FAULT MODEL FOR EXTERNAL SHORTS IN MOS TECHNOLOGIES}

\section{Renovell, P. Huc and Y. Bertrand}

Indexing terms: Integrated circuits, Digital circuits, Logic testing

The Letter focuses on the fault modelling of external shorts in $n-, \mathrm{C}$ - and BiC-MOS digital circuits. In the context of functional testing, it is demonstrated that eight different electrical configurations may appear depending on the topologi$\mathrm{cal}$ and technological parameters of the fault. Therefore ight he wired-OR and wired-AND models, classically used for test pattern generation, fault simulation and defect coverage evaluation are not sufficient.

Introduction: As the density of devices on VLSI chip increases, shorts are expected to become a predominant defect [1-5]. Moreover, it has been demonstrated that more than $85 \%$ of all the likely to occur shorts are shorts between the outputs of different logic gates (external shorts) [6]. The fault model for external shorts is not clearly defined. In fact, even the electrical behaviour of the fault circuit has not been clearly analysed due to the important number of parameters such as MOS technological and topological parameters. In this context, this Letter provides an accurate theoretical analysis of the static behaviour of MOS gates in the presence of external shorts. The detectability of such faults is studied with respect to functional detection techniques (voltage test). The analysis applies to any MOS technology such as nMOS CMOS, BiCMOS ....

In particular, the electrical analysis will show that the fault is detectable as a logic error for a given range of short resistance values. However, in this range, the fault model to be used is independent of the short resistance value. In the case of logically detectable fault, it is demonstrated that eight different cases must be considered. Therefore, eight fault models are defined with a generic name 'dominance'

Electrical analysis: Fig. 1 shows an external short between the outputs $\mathbf{A}$ and $\mathbf{B}$ of two gates. Two detection conditions creating an unwanted conducting path from $V_{D D}$ to GND, are possible. Under condition $1(2), A$ is set to $0(1)$ and $B$ to $1(0)$ Under condition 1 (2), we have $A B=01$ (10) and the potentials are $V_{a}^{01}$ and $V_{b}^{01}\left(V_{a}^{10}\right.$ and $\left.V_{b}^{10}\right)$. It is very important to note that under conditions 1 and 2 the conducting paths are different and the resulting potentials are different: $V_{a}^{01} \neq V_{a}^{10}$ and $V_{b}^{01} \neq V_{b}^{10}$.
Of course, the potentials $V_{a}^{01}, V_{b}^{01}, V_{a}^{10}$ and $V_{b}^{10}$ depend not only on the electrical parameters of the different transistors included in the path, but also on the value $R_{\text {sh }}$ of the short as

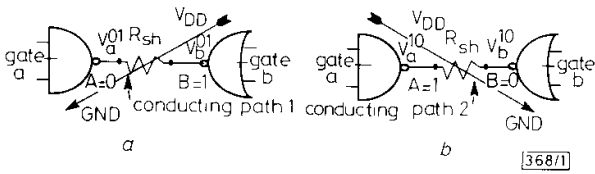

Fig. 1 Two conditions of short detection and corresponding paths a Condition 1

$b$ Condition 2

illustrated in Fig. 2. Three regions are defined according to the intersection with $V_{H}$ and $V_{L}$ : the noise margin of the cells connected to $A$ and $B$. For high $R_{s h}$ values (region 1 ), $V_{a}^{01}$ is recognised as a logic 0 and $V_{b}^{01}$ as a logic 1 . The cell operates correctly and the fault cannot be detected. For intermediate values (region 3), $V_{b}^{01}$ is correct and $V_{a}^{01}$ (between $V_{H}$ and $V_{L}$ ) does not correspond to any logical value. The cell does not operate correctly but the fault is not detectable using functional techniques. Finally, for low values (region 2), $V_{b}^{01}$ is correct and $V_{a}^{01}$ is recognised as a logic 1 . Thus, in this case, the cell exhibits a faulty logical behaviour and the short can be detected using classical functional test techniques.

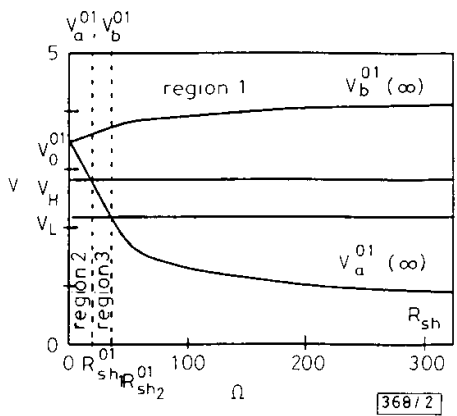

Fig. 2 SPICE simulation of $V_{a}$ and $V_{b}$ against $R_{s h}$ characteristics under condition 1

Logical fault model: In region 2 of Fig. $2, V_{a}^{01}$ is recognised as a logic 1 whatever the value of the short resistance. That implies that the logical behaviour and consequently the faul model are the same whatever the value of the short resistance. Therefore, we can consider a nonresistive short to determine the fault model. Note that the resistance of the short determines the logical detectability of the fault (i.e. the region) bu the fault model to be used is independent of this resistance value.

For a nonresistive short, $V^{01}$ and $V_{b}^{01}$ have the same value called $V_{0}^{01}$. The fault model depends not only on the relative location of $V_{0}^{01}$ (condition 1) but also $V_{0}^{10}$ (condition 2). As previously mentioned $V_{0}^{01}$ and $V_{0}^{10}$ are different due to the different conducting paths. They may be greater than $V_{H}$ smaller than $V_{L}$ or somewhere in between implying nine different cases. Fig. 3 gives the values $A^{*}$ and $B^{*}$ of the shorted nodes for the two conditions of detection: $A B=01$ and $A B=10$. To construct the truth table for each case, we must consider the two other combinations which, of course, do not ensure the detection of the fault: $A B=00$ implying $A^{*}=B^{*}=A=B=0^{*}$ and $A B=11$ implying $A^{*}=B^{*}=$ $A=B=1 *$

It clearly appears that the classical wired-OR and wiredAND models correspond to cases 3 and 7. But they only constitute a subset of all the possible cases: 2 among 8 . Note that case 5 corresponds to the limit when region 3 reaches the 0 resistance short $\left(V_{L}<V_{0}^{10}<V_{H}\right.$ and $\left.V_{L}<V_{0}^{01}<V_{H}\right)$; it is not considered because it is not testable using voltage test techniques. Cases 1 and 9 have been recently reported by some authors [7] and compared to a vote configuration where 